\title{
Global Analysis of Steel Constructions with Semi-Rigid Connections
}

\author{
Srđan ŽIVKOVIĆ, Nenad STOJKOVIĆ, Marija SPASOJEVIĆ-ŠURDILOVIĆ, Marko MILOŠEVIĆ
}

\begin{abstract}
In this paper, original extension of classic deformation method for global elastic analysis of steel construction with semi-rigid connections is developed. The original calculation for steel frame structures with semi-rigid connections as a function of rotational rigidity of connection, a realistic parameter for determination of both tension and deformation fields of connection as well as entire construction, has been derived in detail. Due to its generality, simplicity and straight-forward calculation, the method developed in this paper is convenient for both computational utilization and hand calculations. For introduced rotational rigidity of realistic connections, the expressions for determination of bending moments at ends of semi-rigidly connected elements have been derived as well as conditional equations for determination of deformationunspecified values at static load by first-order theory. The obtained results are illustrated in detail on a numerical example. Their comparison with the results of FEM analysis was provided.
\end{abstract}

Keywords: deformation method; design of steel structures; global analysis; semi-rigid connections

\section{INTRODUCTION}

Over the last several decades, many experimental researches and numerical simulations were conducted with the aim of providing better understanding of realistic behaviour of connections in steel frame structures. Many of them showed that real connections could not be classified as perfectly rigid or pinned, but rather as semirigid or deformable connections [1-4]. On the other hand, the degree of connection rigidity was shown to be of great importance for the moment distribution in the case of prefabricated structures, a type of structures common in steel construction. Even a small degree of connection rigidity could result in increase of the moment in a critical cross section and reduce the load carrying capacity of the entire structure [5-7]. Also, a significant number of researches were focused on the economic advantages that arise with the application of the concept taking into consideration semi-rigid connections in the design of steel frame structures [8-10]. It was shown that possible savings could reach even $20-25 \%$ of the original price of the structure, estimated for the case of perfectly rigid connections.

These facts led to intensive development of methods and procedures based on the existing structural analysis methods (see [11] and [12] and references therein), which enable more realistic modelling of the behaviour of the connections between structural elements. In [13], a method was developed for determining the load capacity as well as end member forces and deformations of frames with partial rigid joint connections, utilizing the direct stiffness method. The connections were modelled as rotational springs attached at the ends of framed members. In [14], analysing the effects of flexibility and eccentricity in the nodal connections of plane frames due to static loading, authors developed a numerical model that includes both nonlinear connection behaviour and geometric nonlinearity of the structure. Unlike the above mentioned methods, where the connections were simulated by means of equivalent "springs" or "fictitious members", in [15] the behaviour of semi-rigid connections was analysed using a method in which the stiffness properties of the individual members with a semi-rigid connection at one or both ends were modified and matrix analysis was applied. In [16], a matrix formulation of the system analysis based on the variational principle was developed. Starting from a base stiffness matrix of a beam exposed to bending, for the case of a member semi-rigidly connected at the ends, stiffness matrix, equivalent load vectors and the consistent mass matrix for a semi-rigidly connected beam were derived using variational procedure.

Most of the above-mentioned methods were developed in a matrix form, which is more convenient for the implementation using computational tools.

In this paper, the existing method of deformation [17] has been extended in order to enable its wider and more comprehensive use in terms of global elastic analysis of steel constructions with semi-rigid connections in the function of rotational rigidity, as a realistic parameter for determination of both tension and deformation fields. The proposed method is relatively simple, since all types of members could be considered as a special case of a member with semi-rigid connections. Furthermore, a closed form equations were obtained and the number of linear algebraic equations required for problem solving is reduced. The method is convenient for both computational utilization and hand calculations.

\section{MATHEMATICAL MODEL OF DEFORMABLE CONNECTION}

The main idea of the method applied in this paper was to express each static and deformation unknown variables as a certain amount of independent deformation variables, which can be determined from the system equilibrium conditions, including realistic rotation rigidity of connections. The application of this method is simple when it comes to straight members, which are most common members in construction. In this section, the theoretical considerations of deformable connections are shortly explained.

In general, a semi-rigid connection between two members enables a certain level of additional displacements in directions of all generalized displacements, which are, for plane frame linear element, relative transversal and longitudinal displacement and sectional rotation at the connection. In most of steel-framed constructions, both transversal and longitudinal connection 
displacements are negligible, compared to sectional rotation at the connection. Thus, the effect of semi-rigid connections can be modelled as elastic spring with rotation rigidity $S_{i(k)}$. This coefficient takes into consideration the influence of the rotational rigidity on the change of the values of static and kinematic variables. Numerical value of rotational rigidity of connection-member $i k$ at the $i$-end (or $k$-end) is determined by the following expression (see [18] and [19]):

$S_{i(k)}=\frac{M_{i(k)}}{\Phi_{i(k)}}$

where $M_{i(k)}$ is bending moment at the connection in node $i$ (or $k$ ); $\Phi_{i(k)}$ is relative rotation of connection in node $i$ (or $k)$.

Rotational rigidity of the connection, in terms of geometry, represents the slope of the tangent on the moment-rotation curve.

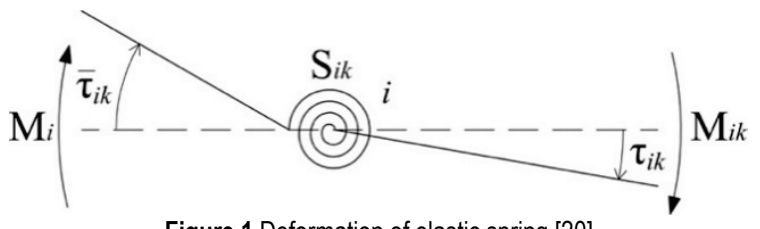

Figure 1 Deformation of elastic spring [20]

Deformation of the connection at node $i$ is represented via elastic spring (Fig. 1), which simulates rotational rigidity $\left(S_{i(k)}\right)$ of the connection, due to unequal rotation of sections both on the left and right side of the connection. On one side, there is rotation of member end (internal spring end) $\tau_{i(k)}$, while on the other there is rotation of the section that includes the node $i$ (external spring end) $\bar{\tau}_{i(k)}$,

and is a function of relative sectional rotation between connection ends $\left(\Phi_{i(k)}=\bar{\tau}_{i(k)}-\tau_{i(k)}\right)$. The elastic spring at the connection between two members must be in equilibrium. Having in mind that $\left(\Phi_{i(k)}=\bar{\tau}_{i(k)}-\tau_{i(k)}\right)$, Eq.

(1) can be rewritten in the following form:

$S_{i k}=\frac{M_{i k}}{\bar{\tau}_{i k}-\tau_{i k}}$

where $M_{i}$ is the bending moment in node $i ; M_{i k}$ is the bending moment at the end $i$ of member $i k ; \tau_{i k}$ is the rotation of cross section of $i k$ member at end $i ; \bar{\tau}_{i k}$ is the rotation in node $i ; \Phi_{i k}$ is relative rotation [21] between the end of member $i k$ and node $i$, as the consequence of the connection deformability.

With the introduction of elastic springs, the idealized member is defined. The ends $i$ and $k$ are elastic springs with rigidity that corresponds to rotational rigidity of realistic connection at the member end, which are connected to other construction elements. The inside end of the spring is connected to the observed member, while the outside end is connected to the node, i.e. the other member of construction. Fig. 2 shows the theoretical model of one member with deformable connections at its ends $i$ and $k$.
Based on the model recommended in [22] as well as many researches listed in [23], the elastic spring can, within tension field and deformation analysis, be considered as infinitely small element which is located at the intersection of system lines of structural members and thereby does not significantly affect the obtained results. This form of idealized member has been implemented in further analysis conducted within this paper.

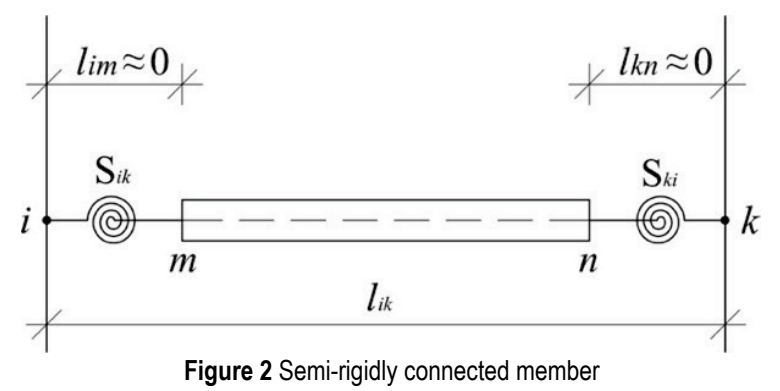

\section{FIRST ORDER THEORY CALCULATION 3.1 Basic Deformation Values of a Straight Member}

Let the member, connected at its ends ( $i$ and $k$ ) to other parts of construction with semi-rigid connections, be separated from the system. Under the effect of arbitrary load $p$ and unknown internal forces at its ends $i$ and $k,\left(N_{i k}\right.$, $T_{i k}, M_{i k}$ and $N_{k i}, T_{k i}, M_{k i}$ ), the member is in state of equilibrium. When the structure deforms, nodes $i$ and $k$ move to the positions $i^{\prime}$ and $k^{\prime}$. Components of nodal displacement in the stationary coordinate system $x O y$ are $u_{i}, v_{i}, u_{k}$ and $v_{k}$. The member $i k$ rotates in such a way that the tendons of undeformed $(i k)$ and deformed $\left(i^{\prime} k^{\prime}\right)$ member form the angle $\psi_{i k}=\psi_{k i}$. Due to the member deformation, its length, $l_{i k}$, becomes length $l_{i k}+\delta l_{i k}=l_{i k} \cdot\left(1+\varepsilon_{i k}\right)$, where $\varepsilon_{i k}$ is its average strain. As the result of the load action, normal to the cross-section of member $i k$ at position $i^{\prime}$ rotates and forms the angle $\tau_{i k}$ with the tendon $i^{\prime} k^{\prime}$. As a result of $i k$ member deformability at end $i^{\prime}$, node $i^{\prime}$ rotates additionally by the angle $\Phi_{i k}$, relative to the tangent of deformed axis of $i k$ member at node $i$. Corresponding deformations also occur at end $k$, also deformably connected to the adjacent member. Thereby, the angle of rotation $\psi_{k i}$ is the consequence of the displacements of member ends and $\Phi_{i k}$ is the consequence of the deformability of member connection at end $k$ as well as the deformation angle of member rotation at node $k\left(\tau_{k i}\right)$.

Angle $\psi_{i k}$ is the member's angle of rotation. This angle does not represent a deformation variable, since it can occur even when the member is not deformed. Hence, it is possible for the member to still rotate as a rigid body, without deforming due to member ends' displacements. Angles $\varphi_{i}$ and $\varphi_{k}$, are the rotation angles of nodes $i$ and $k$, respectively. Angles of deformation, $\bar{\tau}_{i k}$ and $\bar{\tau}_{k i}$ (Figs. 1 and 3$)$, are the result of the deformation of member $i k\left(\tau_{i k}\right)$ and also the consequence of connection deformability at the ends of the member $\left(\Phi_{i k}\right)$. The values of $\bar{\tau}_{i k}$ and $\bar{\tau}_{k i}$ are given by the following equations:

$\bar{\tau}_{i k}=\tau_{i k}+\Phi_{i k}$
$\bar{\tau}_{k i}=\tau_{k i}+\Phi_{k i}$ 
Deformation angles of member $i k\left(\tau_{i k}\right.$ and $\left.\tau_{k i}\right)$ can be formulated for the load $p$, temperature difference $\Delta t^{\circ}$ and bending moments at the member ends $M_{i k}$ and $M_{k i}$. The rotation of ends of member due to elastic deformation of connections $\left(\Phi_{i(k)}\right)$ can be expressed with function of rotational rigidity $\left(S_{i(k)}\right)$ of those connections and bending moments at the member's ends $\left(M_{i k}\right.$ and $\left.M_{k i}\right)$ :

$$
\begin{gathered}
\Phi_{i k}=\frac{M_{i k}}{S_{i k}} \\
\Phi_{k i}=\frac{M_{k i}}{S_{k i}}
\end{gathered}
$$

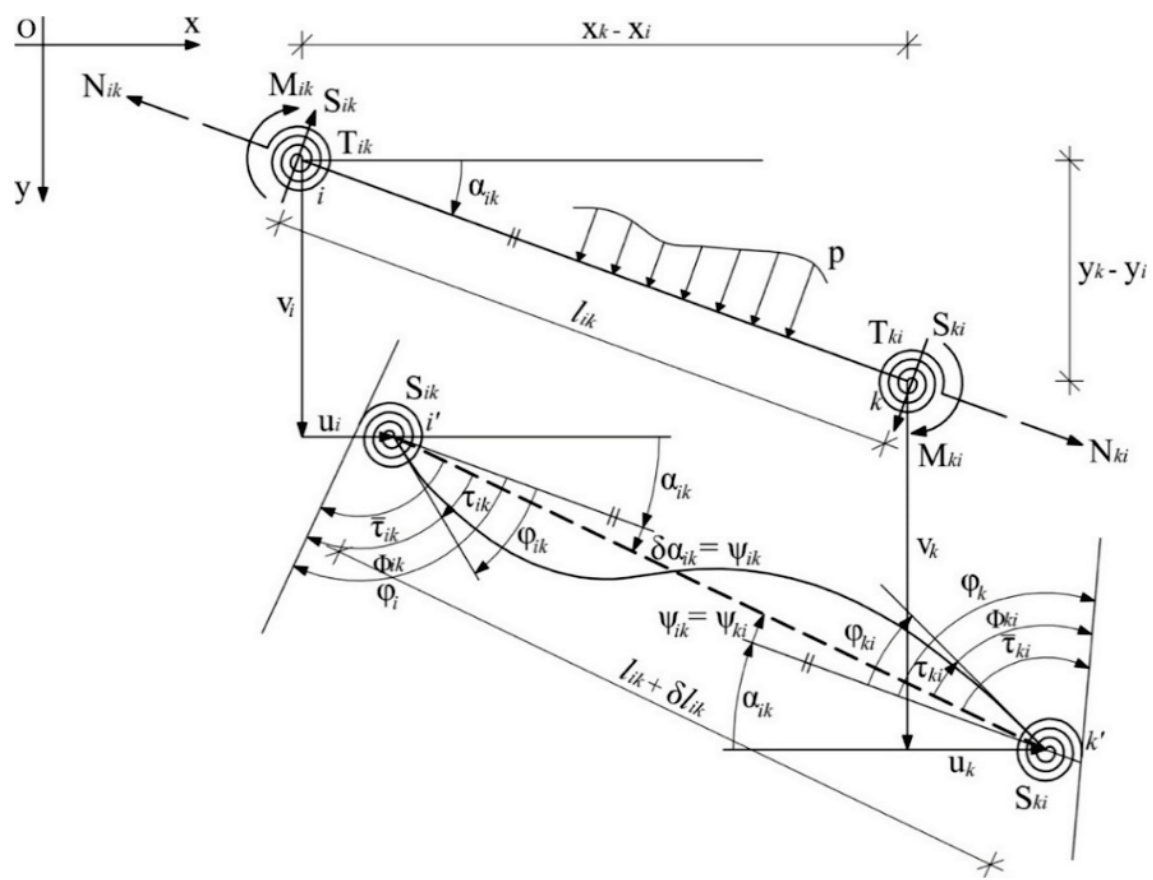

Figure 3 Straight semi-rigidly connected member at its ends (before and after deformation)

Due to connection deformability, the rotations of member ends $\left(\varphi_{i k}\right.$ and $\left.\varphi_{k i}\right)$, are not equal to the rotation of nodes $\left(\varphi_{i}\right.$ and $\left.\varphi_{k}\right)$, which is shown in Fig. 3. The difference between them is given by the following equations:

$\varphi_{i}-\varphi_{i k}=\bar{\tau}_{i k}-\tau_{i k}=\Phi_{i k}$

$\varphi_{k}-\varphi_{k i}=\bar{\tau}_{k i}-\tau_{k i}=\Phi_{k i}$

i.e.

$\bar{\tau}_{i k}=\tau_{i k}+\Phi_{i k}=\varphi_{i}-\psi_{i k}$

$\bar{\tau}_{k i}=\tau_{k i}+\Phi_{k i}=\varphi_{k}-\psi_{i k}$

The notation is shown in Fig. 4. The load induced rotation of the elastic line tangents at the ends $i$ and $k$ of the simple beam are denoted by $\alpha_{i k}^{(0)}$ and $\alpha_{k i}^{(0)}$. Further, $\alpha_{i k}^{(\Delta t)}$ and $\alpha_{k i}^{(\Delta t)}$ are the corresponding angles caused by the effect of the temperature difference $(\Delta t)$, and $\alpha_{i k}$ and $\beta_{i k}\left(\alpha_{k i}\right.$ and $\beta_{k i}$ ) are the rotation angles that emerge as the effect of unit bending moments $M_{i k}=1$ and $M_{k i}=1$. Substituting Eq. (4) into Eq. (6) and applying the principle of superposition, the following expressions are obtained:

$$
\begin{aligned}
& \alpha_{i k}^{(0)}+\alpha_{i k}^{(\Delta t)}+M_{i k} \cdot \alpha_{i k}-M_{k i} \cdot \beta_{i k}+\frac{M_{i k}}{S_{i k}}=\varphi_{i}-\psi_{i k}, \\
& -\alpha_{k i}^{(0)}-\alpha_{k i}^{(\Delta t)}-M_{i k} \cdot \beta_{k i}+M_{k i} \cdot \alpha_{k i}+\frac{M_{k i}}{S_{k i}}=\varphi_{k}-\psi_{i k} .
\end{aligned}
$$
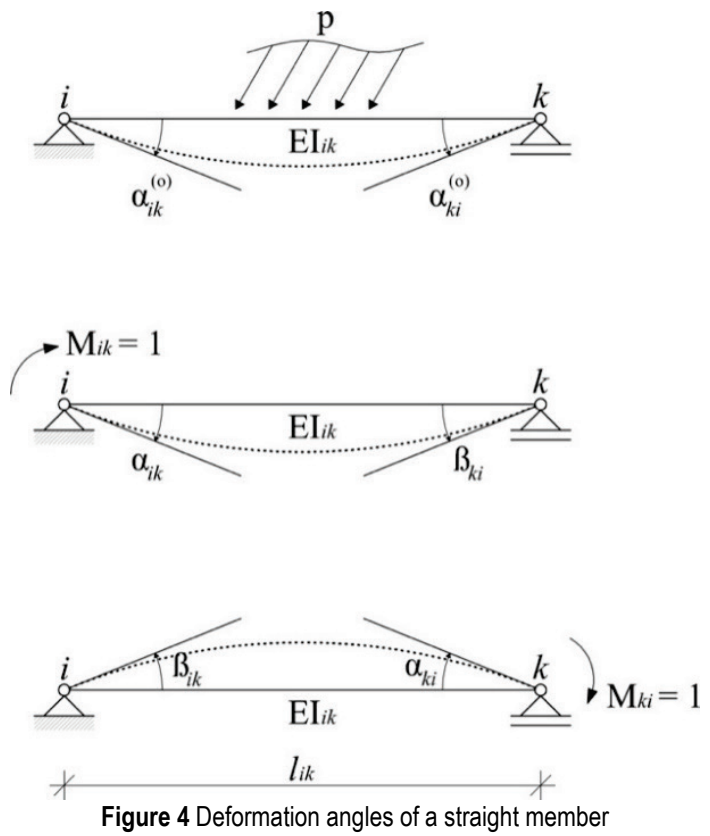

Based on Maxwell's theorem, the angles $\beta_{i k}$ and $\beta_{k i}$ are equal. On the other hand, in general case, $\alpha_{i k} \neq \alpha_{k i}$ for $E I \neq$ const. If the load $p$ and the temperature changes $\Delta t$ are known variables, the values $\alpha_{i k}^{(0)}, \alpha_{k i}^{(0)}, \alpha_{i k}^{(\Delta t)}, \alpha_{k i}^{(\Delta t)}$, $\alpha_{i k}, \alpha_{k i}, \beta_{i k}$ and $\beta_{k i}$ can be calculated for the effect of unit bending moments $M_{i k}=1$ and $M_{k i}=1$. Eq. (7) can be rewritten as follows: 


$$
\begin{aligned}
& M_{i k} \cdot\left(\alpha_{i k}+\frac{1}{S_{i k}}\right)-M_{k i} \cdot \beta_{i k}=\varphi_{i}-\psi_{i k}-\alpha_{i k}^{(o)}-\alpha_{i k}^{(\Delta t)}, \\
& -M_{i k} \cdot \beta_{k i}-M_{k i} \cdot\left(\alpha_{k i}+\frac{1}{S_{k i}}\right)=\varphi_{k}-\psi_{i k}-\alpha_{k i}^{(o)}-\alpha_{k i}^{(\Delta t)} .
\end{aligned}
$$

From Eq. (8), the following expressions are obtained for $M_{i k}$ and $M_{k i}$.

$$
\begin{aligned}
M_{k i}= & M_{i k}=\frac{\alpha_{k i}+\frac{1}{S_{k i}}}{D} \cdot\left(\varphi_{i}-\psi_{i k}-\alpha_{i k}^{(0)}-\alpha_{i k}^{(\Delta t)}\right)+ \\
& +\frac{\beta_{i k}}{D} \cdot\left(\varphi_{k}-\psi_{i k}+\alpha_{k i}^{(0)}+\alpha_{k i}^{(\Delta t)}\right) \\
M_{k i}= & \frac{\alpha_{i k}+\frac{1}{S_{i k}}}{D} \cdot\left(\varphi_{k}-\psi_{i k}+\alpha_{k i}^{(0)}+\alpha_{i k}^{(\Delta t)}\right)+ \\
& +\frac{\beta_{i k}}{D} \cdot\left(\varphi_{i}-\psi_{i k}-\alpha_{i k}^{(0)}-\alpha_{k i}^{(\Delta t)}\right)
\end{aligned}
$$

Parameter $D$ is expressed as:

$D=\left|\begin{array}{cc}\alpha_{i k}+\frac{1}{S_{i k}} & -\beta_{i k} \\ -\beta_{i k} & \alpha_{k i}+\frac{1}{S_{k i}}\end{array}\right|=\left(\alpha_{i k}+\frac{1}{S_{i k}}\right) \cdot\left(\alpha_{k i}+\frac{1}{S_{k i}}\right)-\beta_{i k}^{2}$

where:

$\bar{a}_{i k}=\frac{\alpha_{k i}+\frac{1}{S_{k i}}}{D}, \quad \bar{a}_{k i}=\frac{\alpha_{i k}+\frac{1}{S_{i k}}}{D}$

$\bar{b}_{i k}=\frac{\beta_{i k}}{D}=\bar{b}_{k i}$

$\bar{c}_{i k}=\bar{a}_{i k}+\bar{b}_{i k}, \quad \bar{c}_{k i}=\bar{a}_{k i}+\bar{b}_{k i}$

$\bar{m}_{i k}^{(o)}=-\left(\bar{a}_{i k} \cdot \alpha_{i k}^{(0)}-\bar{b}_{i k} \cdot \alpha_{k i}^{(0)}\right), \bar{m}_{k i}^{(0)}=\bar{a}_{k i} \cdot \alpha_{k i}^{(0)}-\bar{b}_{i k} \alpha_{i k}^{(0)}$

$\bar{m}_{i k}^{(\Delta t)}=-\left(\bar{a}_{i k} \cdot \alpha_{i k}^{(\Delta t)}-\bar{b}_{i k} \cdot \alpha_{k i}^{(\Delta t)}\right), \bar{m}_{k i}^{(\Delta t)}=\bar{a}_{k i} \cdot \alpha_{k i}^{(\Delta t)}-\bar{b}_{i k} \alpha_{i k}^{(\Delta t)}$

From Eqs. (9), (10) and (12) to (16), expressions for moments at ends $i$ and $k$ of member $i k$ can be formulated as:

$$
\begin{aligned}
& M_{i k}=\bar{a}_{i k} \cdot \varphi_{i}+\bar{b}_{i k} \cdot \varphi_{k}-\bar{c}_{i k} \cdot \psi_{i k}+\bar{m}_{i k}^{(0)}+\bar{m}_{i k}^{(\Delta t)}, \\
& M_{k i}=\bar{b}_{i k} \cdot \varphi_{i}+\bar{a}_{k i} \cdot \varphi_{k}-\bar{c}_{k i} \cdot \psi_{i k}+\bar{m}_{k i}^{(0)}+\bar{m}_{k i}^{(\Delta t)} .
\end{aligned}
$$

In general, for prismatic member $E I_{i k}=$ const., the values of Eq. (17) satisfy the conditions $\bar{a}_{i k} \neq \bar{a}_{k i}, \bar{b}_{i k} \neq \bar{b}_{k i}$ and $\bar{c}_{i k} \neq \bar{c}_{k i}$. The reason for this is that values $\bar{a}_{i k}, \bar{b}_{i k}, \bar{c}_{i k}, \bar{a}_{k i}, \bar{b}_{k i}, \bar{c}_{k i}$ depend not only on the geometrical properties of the cross section and mechanical properties of primary material, but also on characteristics of connections at member ends (the constants of the semi-rigidly connected member).

Physical representation of certain values, denoted as in Fig. 5, can be explained by analyzing Eq. (17):

- $\bar{a}_{i k}$ and $\bar{b}_{i k}$ represent bending moments of semirigidly connected member at ends $i$ and $k\left(M_{i k}\right.$ and $\left.M_{k i}\right)$ when

$\varphi_{i}=1$ and $\varphi_{k}=\psi_{i k}=\bar{m}_{i k}^{(0)}=\bar{m}_{k i}^{(0)}=\bar{m}_{i k}^{(\Delta t)}=\bar{m}_{k i}^{(\Delta t)}=0$;

- $\quad \bar{c}_{i k}$ and $\bar{c}_{k i}$ represent bending moments of semi-rigidly connected member at ends $i$ and $k\left(M_{i k}\right.$ and $\left.M_{k i}\right)$ when $\psi_{i k}=-1$ and $\varphi_{i}=\varphi_{k}=\psi_{i k}=\bar{m}_{i k}^{(0)}=\bar{m}_{k i}^{(0)}=\bar{m}_{i k}^{(\Delta t)}=\bar{m}_{k i}^{(\Delta t)}$ $=0$;

- $\bar{m}_{i k}^{(0)}, \bar{m}_{k i}^{(0)}, \bar{m}_{i k}^{(\Delta t)}$ and $\bar{m}_{k i}^{(\Delta t)}$ represent bending moments of semi-rigidly connected member member at ends $i$ and $k \quad\left(M_{i k}\right.$ and $\left.M_{k i}\right)$ when $\psi_{i k}=0$, $\varphi_{1}=0 \Rightarrow \tau_{i k}=-\Phi_{i k}$ and $\varphi_{k i}=0 \Rightarrow \tau_{k i}=-\Phi_{k i}$, due to given load $p$ and temperature difference $\Delta t$.

(a)
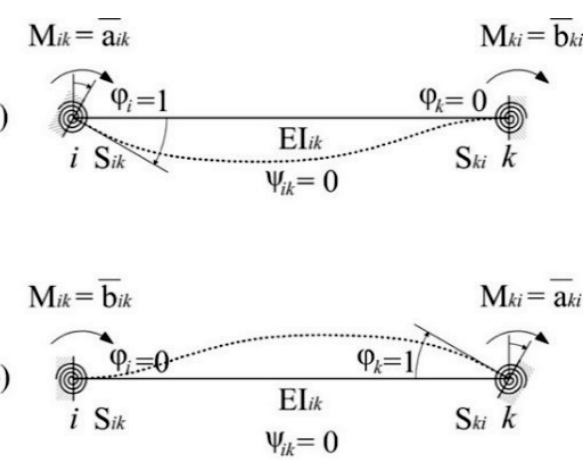

(c)

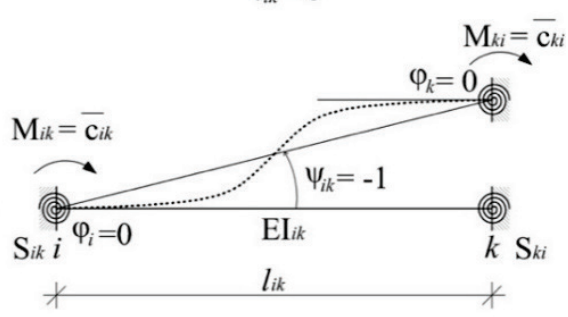

(d)

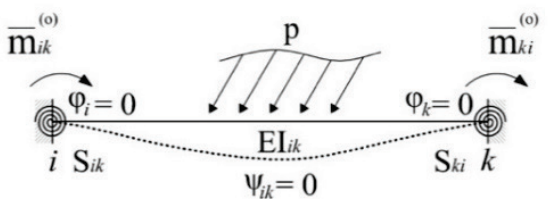

Figure 5 Physical representation of member constants and initial bending moments of semi-rigidly connected member

Within Eqs. (12) to (16), the values of rotational rigidity at member ends $\left(S_{i k}\right.$ and $\left.S_{k i}\right)$ are determined by calculations procedures given in [6].

In the case of a member rigidly connected at both its ends $\left(S_{i k}, S_{k i} \rightarrow \infty\right)$, the obtained values yield to values of $k$ type member, defined within classical method of deformation [17]. If the member $i k$ has rigid connection at its end $i$ and pinned at its end $k\left(S_{i k} \rightarrow \infty\right.$ and $\left.S_{k i} \rightarrow 0\right)$, the obtained values converge to values of type $g$ member, defined within classic method of deformation. For the case of $i k$ member with pinned connections at both ends, $\left(S_{i k}, S_{k i}\right.$ $\rightarrow 0$ ), the obtained values converge to obtained values yield to values of $s$-type member, also defined within 
classic method of deformation. The previous results imply that all types of members can be treated as a universal member type with semi-rigid connections, which simplifies and unifies the calculation to a large extent. This fact is specifically significant for the implementation of the developed equations in the structural analysis software.

\subsection{Conditional Equations for Unknown Variables}

If the influence of normal forces on the deformation is neglected (the theory known as approximate method of deformation), the indeterminacy of the system significantly decreases, which makes this method more convenient. Even though the introduced assumption of neglecting the effect of normal forces on structural deformation in most cases has negligible effect, in the case of special loads or very tall objects it can result in significant errors. The possibility of increasing the accuracy of approximate method of deformation up to the required level, in cases when it is necessary, is discussed in publications [11] and [24]. It can be also applied within presented method. Bending moments at member ends can be expressed as a function of node rotation $\varphi_{i}$ and kinematic parameter $\Delta j$, which is equal to the "sway" of the $j$-th fictious member of the substituting truss (for more details see [4]):

$$
\begin{aligned}
& M_{i k}=\bar{a}_{i k} \cdot \varphi_{i}+\bar{b}_{i k} \cdot \varphi_{k}-\bar{c}_{i k} \cdot \sum_{j=1}^{n} \psi_{i k}^{(j)} \cdot \Delta_{j}+\bar{m}_{i k}, \\
& M_{k i}=\bar{b}_{i k} \cdot \varphi_{i}+\bar{a}_{k i} \cdot \varphi_{k}-\bar{c}_{k i} \cdot \sum_{j=1}^{n} \psi_{i k}^{(j)} \cdot \Delta_{j}+\bar{m}_{k i}
\end{aligned}
$$

where:

$$
\begin{aligned}
\bar{m}_{i k} & =\bar{m}_{i k}^{(0)}+\bar{m}_{i k}^{(\Delta t)}+\bar{m}_{i k}^{(t)}+\bar{m}_{i k}^{(c)}= \\
& =\bar{m}_{i k}^{(0)}+\bar{m}_{i k}^{(\Delta t)}-c_{i k} \psi_{i k}^{(t)}-c_{i k} \psi_{i k}^{(c)}, \\
\bar{m}_{k i} & =\bar{m}_{k i}^{(0)}+\bar{m}_{k i}^{(\Delta t)}+\bar{m}_{k i}^{(t)}+\bar{m}_{k i}^{(c)}= \\
& =\bar{m}_{k i}^{(0)}+\bar{m}_{k i}^{(\Delta t)}-c_{k i} \psi_{k i}^{(t)}-c_{k i} \psi_{k i}^{(c)} .
\end{aligned}
$$

Based on Eqs. (18) and (19), it can be concluded that shear forces and bending moments can be expressed using the same deformational independent values. The first group of equations, as in the classic method of deformation, represents the equilibrium condition for bending moments in node $i$ :

$$
\sum_{k=1}^{r} M_{i k}+M_{i}=0, \quad(i=1,2, \ldots, m)
$$

where $\sum_{k=1}^{r} M_{i k}$ is the sum of bending moments of all members intersecting in node $i ; M_{i}$ is the sum of all bending moments of cantilever members at node $i$, which do not depend on member deformation and enter the calculation as already known value, $m$ is the number of nodes in which at least two members are connected; and $r$ is the number of members connected in observed node $i$.
Eq. (20) can be written for each node of the system in which there is at least one member with semi-rigid connection, giving the total number of $m$ equations. Substituting Eq. (18) into Eq. (20) and applying basic mathematic transformations, these equations can be expressed as:

$$
\bar{A}_{i i} \cdot \varphi_{i}+\sum_{k=1}^{r} \bar{A}_{i k} \cdot \varphi_{k}+\sum_{j=1}^{n} \bar{B}_{i j} \cdot \Delta_{j}+\bar{A}_{i o}=0,(i=1, \ldots, m)
$$

with notation:

$$
\begin{aligned}
& \bar{A}_{i i}=\sum_{k} \bar{a}_{i k} ; \quad \bar{A}_{i k}=\bar{b}_{i k} ; \quad \bar{A}_{i o}=\sum_{k} \bar{m}_{i k}+M_{i} \\
& \bar{B}_{i j}=-\sum_{k} \bar{c}_{i k} \cdot \psi_{i k}^{(j)}
\end{aligned}
$$

The second group of equations represents the conditions under which the substituting system truss, subjected to external forces which are including the moments at the member ends, is in equilibrium. According to the principle of virtual displacements, for one kinematically unstable system to be in equilibrium it is necessary to keep the sum of work of external forces that affect the system, under any given virtual movement, equal to zero. Denoting $R j$ as the work of external forces under the "sway" $\Delta j=-1$, these conditions can be expressed for each possible state of virtual displacement $(j=1,2, \ldots, n)$ :

$$
-\sum_{i k}\left(M_{i k}+M_{k i}\right) \cdot \psi_{i k}^{(j)}-R_{j}=0, \quad(j=1,2, \ldots, n)
$$

where $\sum_{i k}$ is the sum taken over all members. Substituting Eq. (18) into Eq. (24) and applying basic mathematical transformations, these equations can be written as:

$$
\sum_{i=1}^{m} \bar{B}_{j i}^{\prime} \cdot \varphi_{i}+\sum_{l=1}^{n} \bar{C}_{j l} \cdot \Delta_{l}+\bar{C}_{j o}=0, \quad(j=1,2, \ldots, n)
$$

where:

$$
\begin{aligned}
\bar{B}_{j i}^{\prime} & =-\sum_{k} \bar{c}_{i k} \cdot \psi_{i k}^{(j)}=\bar{B}_{i j} \\
\bar{C}_{j l} & =\sum_{i k}\left(\bar{c}_{i k}+\bar{c}_{k i}\right) \cdot \psi_{i k}^{(j)} \cdot \psi_{i k}^{(j)} ; \bar{C}_{j o}= \\
& =-\sum_{i k}\left(\bar{m}_{i k}+\bar{m}_{k i}\right) \cdot \psi_{i k}^{(j)}-R_{j}
\end{aligned}
$$

In such a way, a system of $m+n$ linear algebraic equations can be obtained, which can be used to determine $m$ values of $\varphi_{i}$ and $n$ values of $\Delta j$.

\section{DETERMINATION OF CONSTANTS AND BENDING MOMENTS AT THE ENDS OF PRISMATIC MEMBERS WITH SEMI-RIGID CONNECTIONS}

In this section, the equations for determining the constants and bending moments at the ends of members are derived for the case of homogeneous, isotropic and ideally 
elastic prismatic member. For members that have constant rigidity, which is a common case in construction, it is possible to simplify the procedure of determining constants and bending moments of members with semi-rigid connections. It is known that the constants of a member with ideally-rigid connections are $[23,25]$ :

$$
a_{i k}=a_{k i}=\frac{4 E I_{i k}}{l_{i k}} ; b_{i k}=b_{k i}=\frac{2 E I_{i k}}{l_{i k}} ; c_{i k}=c_{k i}=\frac{6 E I_{i k}}{l_{i k}}
$$

For a semi-rigidly connected member with rotational rigidities $S_{i k}$ and $S_{k i}$ at nodes $i$ and $k$, respectively, new dimensionless parameters of realistic rigidity at member ends $i$ and $k$ can be introduced:

$\Psi_{i k}=\frac{E I_{i k}}{l \cdot S_{i k}}$ and $\Psi_{k i}=\frac{E I_{i k}}{l \cdot S_{k i}}$

Member constants and bending moments of members with semi-rigid connections are determined through their physical significance, as defined in section 3 . In order to determine the constants of members, the well-known force method $[11,20]$ is applied, and the following equations are derived:

$$
\begin{aligned}
& \bar{a}=a \cdot \eta \\
& \bar{a}_{k i}=a_{k i} \cdot \eta_{3} \\
& \bar{b}_{k i}=\bar{b}_{i k}=b_{k i} \cdot \eta_{2}=b_{i k} \cdot \eta_{2} \\
& \bar{c}_{i k}=c_{i k} \cdot \eta_{4} \\
& \bar{c}_{k i}=c_{k i} \cdot \eta_{5} \\
& \Delta=1+4\left(\Psi_{i k}+\Psi_{k i}\right)+12 \Psi_{i k} \Psi_{k i} \\
& \eta_{1}=\frac{1+3 \Psi_{k i}}{1+4\left(\Psi_{i k}+\Psi_{k i}\right)+12 \Psi_{i k} \Psi_{k i}}=\frac{1+3 \Psi_{k i}}{\Delta} \\
& \eta_{2}=\frac{1}{1+4\left(\Psi_{i k}+\Psi_{k i}\right)+12 \Psi_{i k} \Psi_{k i}}=\frac{1}{\Delta} \\
& \eta=\frac{1+3 \Psi}{1+4(\Psi+\Psi)+12 \Psi \Psi}=\frac{1+3 \Psi}{\Delta} \\
& \eta_{4}=\frac{1+2 \Psi_{k i}}{\Delta} \\
& \eta_{5}=\frac{1+2 \Psi_{i k}}{\Delta}
\end{aligned}
$$

The following relations between constants have been noticed:

$$
\begin{aligned}
& 2 \eta+\eta=3 \eta \text { and } \\
& 2 \eta_{1}+\eta_{2}=3 \eta_{4}
\end{aligned}
$$

For symmetrical boundary conditions, replacing the rotational rigidities $S_{i k}$ and $S_{k i}$ with $S$, as well as $\Psi_{i k}$ and
$\Psi_{i k}$ with $\Psi$, the coefficients a of a member, semi-rigidly connected at both ends, will have the following values:

$$
\begin{aligned}
& \eta_{1}=\eta_{3}=\frac{1+3 \Psi}{1+8 \Psi+12 \Psi^{2}} \\
& \eta_{2}=\frac{1}{1+8 \Psi+12 \Psi^{2}} \\
& \eta_{4}=\eta_{5}=\frac{1+2 \Psi}{1+8 \Psi+12 \Psi^{2}}
\end{aligned}
$$

The initial bending moments are determined for realistic boundary conditions. Hence, the initial bending moments are calculated using rotational rigidities $S_{i k}$ and $S_{k i}$. The force-method is applied in solving this problem.

For member with unsymmetrical boundary conditions, subjected to uniform load, bending moments of semirigidly connected member can be determined by the procedure given bellow.

For symmetrical boundary conditions, performing a simple replacement $S_{i k}=S_{k i}=S$ and $\Psi_{i k}=\Psi_{k i}=\Psi$, the coefficients for semi-rigidly connected member at both ends will have the values:

$\bar{m}_{i k}^{(0)}=\bar{m}_{k i}^{(0)}=m_{i k}^{(0)} \cdot \frac{1+6 \Psi}{\Delta}=m_{k i}^{(0)} \cdot \frac{1+6 \Psi}{\Delta}$

Values of bending moments of semi-rigidlyconnected member given in Eq. (46) can only be determined this way if the load is symmetric, i.e. if $\bar{m}_{i k}^{(0)}=-\bar{m}_{k i}^{(0)}$, which is a common case. Otherwise, general expressions (15) and (16) have to be utilized.

\section{NUMERICAL EXAMPLE AND DISCUSSION}

In order to illustrate the developed method for the analysis of plane frame structure with semi-rigid connections and compare the obtained results with the results of FEM calculation, the analysis was conducted on the plane frame shown in Fig. 6, subjected to loading cases presented in Fig. 7.

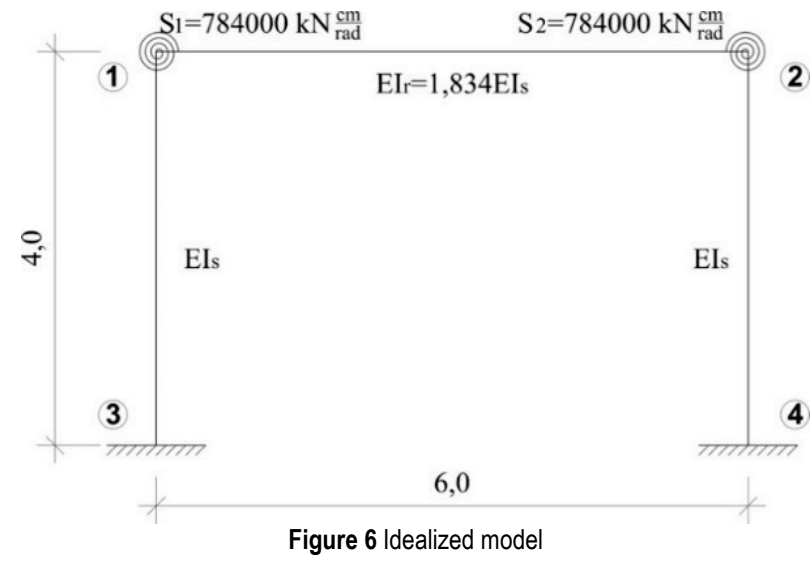

The beam-to-column connections are symmetrical, realized using overhanging end-plate. The rotational 
rigidity of connection is determined according to recommendations given in EC3 [18].

Secant rotational rigidity of connection was $S=$ $784000 \mathrm{kNcm} / \mathrm{rad}$. Member 1-2 (beam) is made of hot rolled steel profile IPE 220, with the moment of inertia $I_{x, r}$ $=2770,0 \mathrm{~cm}^{4}$. Members 1-3 and 2-4 (columns) are made of hot rolled steel profiles HE-B 140, with the moment of inertia $I_{x, s}=1510,0 \mathrm{~cm}^{4}$.

Two loading cases are considered:

a) symmetrical uniform vertical load (Fig. 7a) and

b) horizontal load (Fig. 7b).
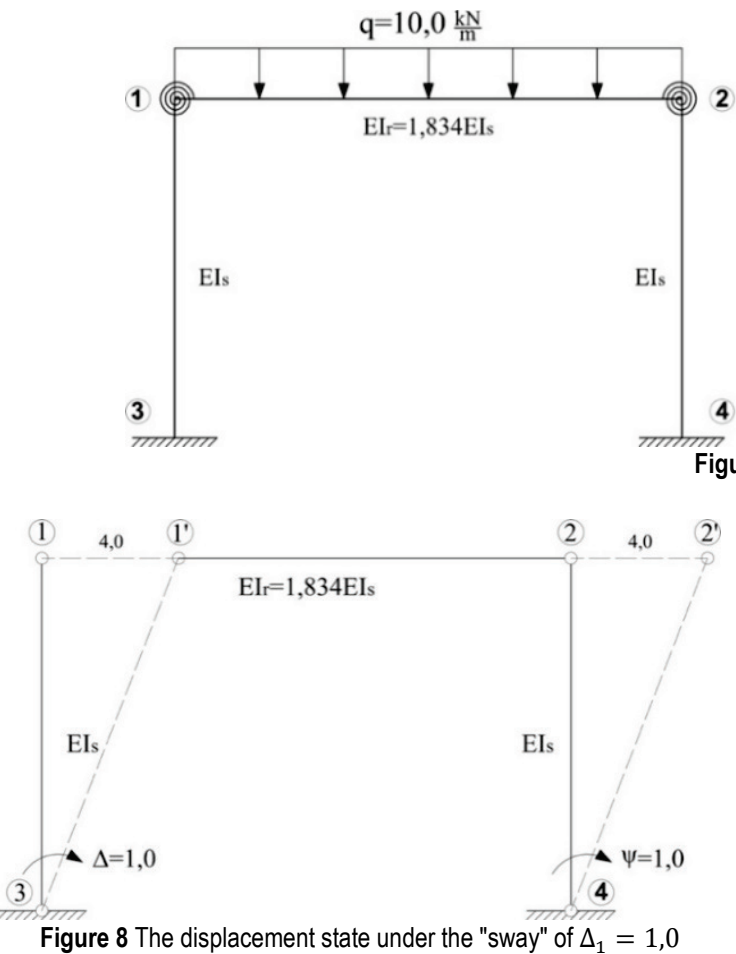

Rigidly connected member constants at both sides, according to Eq. (28), are:

$$
\begin{aligned}
& a_{12}=a_{21}=1,223 E I_{s} \\
& a_{13}=a_{31}=E I_{s} \\
& a_{24}=a_{42}=E I_{s} \\
& b_{12}=b_{21}=0,611 E I_{s} \\
& b_{13}=b_{31}=0,5 E I_{s} \\
& b_{24}=b_{42}=0,5 E I_{s} \\
& c_{12}=c_{21}=1,834 E I_{s} \\
& c_{13}=c_{31}=1,5 E I_{s} \\
& c_{24}=c_{42}=1,5 E I_{s} .
\end{aligned}
$$

Eq. (46) gives initial bending moments for rigidly connected member $m_{12}^{(0)}=-30,0 \mathrm{kNm} ; m_{21}^{(0)}=30,0 \mathrm{kNm}$.

Parameters of rigidity and reduction coefficients, according to Eqs. (29) to (40) are as follows:

\section{Member 1-2}

$\Psi_{1}=\Psi_{2}=0,124, \Delta=2,177 ; \eta_{1}=0,630 ; \eta_{2}=0,459$;

$\eta_{3}=0,630 ; \eta_{4}=0,573 ; \eta_{5}=0,573$.
The system is three times indeterminate. Angles of rotation $\left(\varphi_{1}, \varphi_{2}\right)$, as well as kinematic parameter of the substituting truss $\left(\Delta_{1}\right)$, are unknown. The displacement state under the "sway" of $\Delta_{1}=1,0$ is shown in Fig. 8.

Conditional Eqs. (21) and (25) for determination of unknown values can be expressed in matrix form as:

$$
\left[\begin{array}{lll}
\bar{A}_{11} & \bar{A}_{12} & \bar{B}_{11} \\
\bar{A}_{21} & \bar{A}_{22} & \bar{B}_{21} \\
\bar{B}_{11}^{\prime} & \bar{B}_{12}^{\prime} & \bar{C}_{11}
\end{array}\right] \cdot\left\{\begin{array}{l}
\varphi_{1} \\
\varphi_{2} \\
\Delta_{1}
\end{array}\right\}+\left\{\begin{array}{l}
\bar{A}_{10} \\
\bar{A}_{20} \\
\bar{C}_{10}
\end{array}\right\}=\left\{\begin{array}{l}
0 \\
0 \\
0
\end{array}\right\}
$$

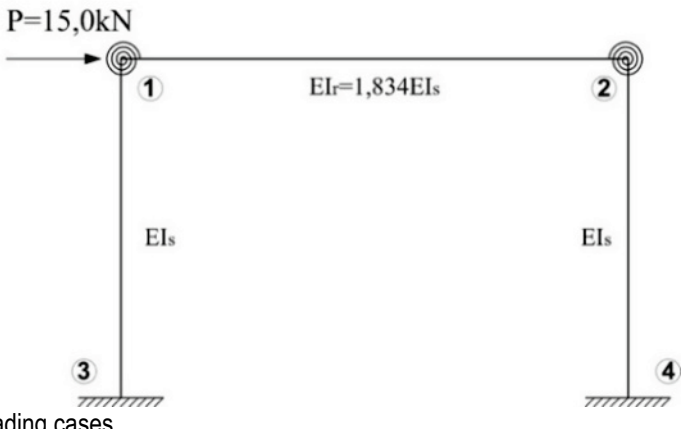

gure 7 Loading cases 
Eq. (27) gives the value of $\bar{C}_{10}=0$.

The obtained system of equation, given in a matrix form, is:

$\left[\begin{array}{ccc}1,482 & 0,280 & -1,068 \\ 0,280 & 1,482 & -1,068 \\ -1,068 & -1,068 & 4,704\end{array}\right] \cdot\left\{\begin{array}{l}\varphi_{1} \\ \varphi_{2} \\ \Delta_{1}\end{array}\right\}+\left\{\begin{array}{c}-24,033 / E I_{s} \\ +24,033 / E I_{s} \\ 0\end{array}\right\}=\left\{\begin{array}{l}0 \\ 0 \\ 0\end{array}\right\}$

Solutions of the system of equations are:

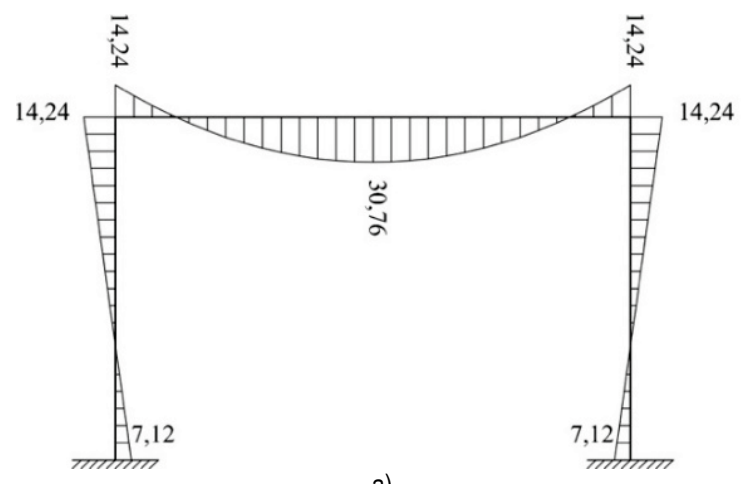

Figure 9 Bending moment diagrams in $\mathrm{kNm}$ - loading case l: a) semi-rigid connections at nodes 1 and 2; b) rigid connections

$$
\begin{aligned}
& \varphi_{1}=\frac{19,9942}{E I_{s}} \\
& \varphi_{2}=-\frac{19,9942}{E I_{s}} \\
& \Delta_{1}=\frac{-2,06072 \cdot 10^{-16}}{E I_{s}} \approx 0 .
\end{aligned}
$$

Moments at ends of deformable connected member, calculated using Eq. (18), are shown in Fig. 9.

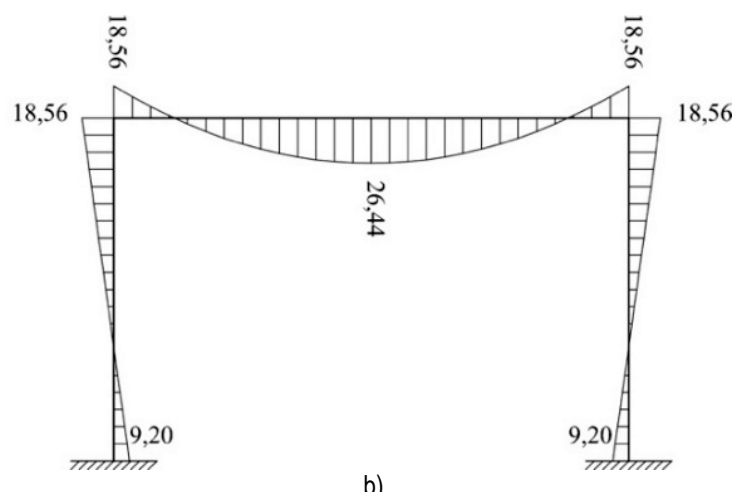

\subsection{Loading case II}

Eq. (22) gives independent coefficients $\bar{A}_{10}=0$ $\bar{A}_{20}=0$ and Eq. (27) gives that $\bar{C}_{10}=-60 \mathrm{kNm}$.

Conditional equations of approximate deformation method, given in a matrix form, are:

$$
\left[\begin{array}{ccc}
1,482 & 0,280 & -1,068 \\
0,280 & 1,482 & -1,068 \\
-1,068 & -1,068 & 4,704
\end{array}\right] \cdot\left\{\begin{array}{l}
\varphi_{1} \\
\varphi_{2} \\
\Delta_{1}
\end{array}\right\}+\left\{\begin{array}{c}
0 \\
0 \\
-60,0 / E I_{s}
\end{array}\right\}=\left\{\begin{array}{l}
0 \\
0 \\
0
\end{array}\right\}
$$

芒

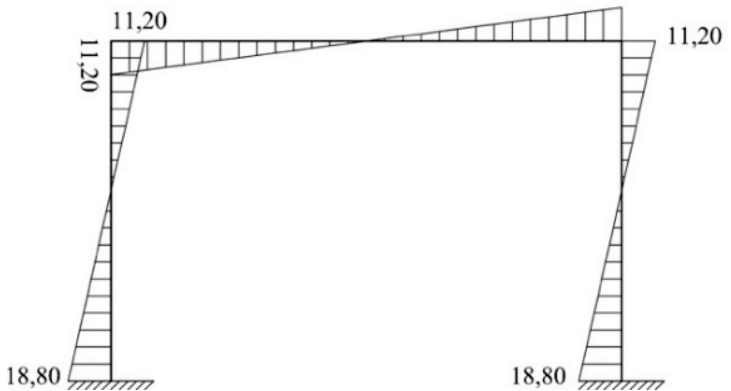

a)

Figure 10 Bending moment diagrams in $\mathrm{kNm}$ - loading case II: a) semi-rigid connections at nodes 1 and 2; b) rigid connections
Solutions of conditional equations are:

$$
\begin{gathered}
\varphi_{1}=\frac{10,6672}{E I_{s}} \\
\varphi_{2}=\frac{10,6672}{E I_{s}}
\end{gathered}
$$$$
\Delta_{1}=\frac{17,5989}{E I_{s}}
$$

Moments at ends of deformable connected member, calculated using Eq. (47), are shown in Fig. 10.

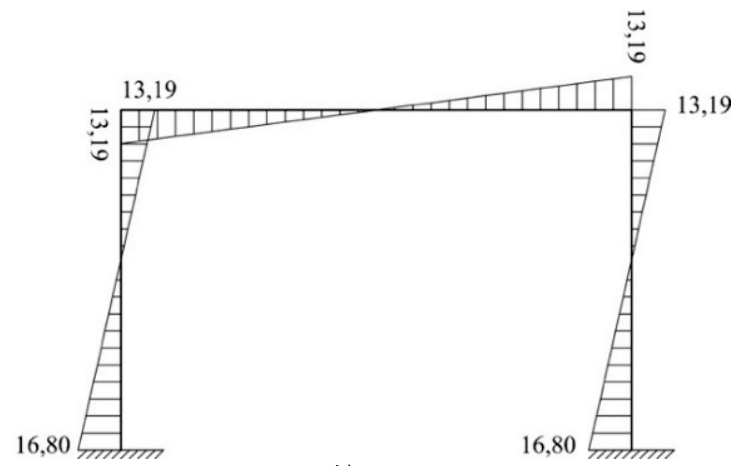

$\vec{\omega}$
Table 1 The results for loading case I

\begin{tabular}{|c|c|c|c|}
\hline Source & $\begin{array}{c}\text { Moment at } \\
\text { field / kNm }\end{array}$ & $\begin{array}{c}\text { Moment at } \\
\text { connection / kNm }\end{array}$ & $\begin{array}{c}\text { Moment at } \\
\text { backbone / kNm }\end{array}$ \\
\hline FEM [24] & 30,79 & 14,21 & 7,11 \\
\hline $\begin{array}{c}\text { Method } \\
\text { developed in } \\
\text { this paper }\end{array}$ & 30,76 & 14,24 & 7,12 \\
\hline
\end{tabular}

Table 2 The results for loading case II

\begin{tabular}{|c|c|c|c|}
\hline Source & $\begin{array}{c}\text { Moment at } \\
\text { field } / \mathrm{kNm}\end{array}$ & $\begin{array}{c}\text { Moment at } \\
\text { connection } / \mathrm{kNm}\end{array}$ & $\begin{array}{c}\text { Moment at } \\
\text { backbone } / \mathrm{kNm}\end{array}$ \\
\hline FEM [24] & 0 & 11,19 & 18,81 \\
\hline $\begin{array}{c}\text { Method } \\
\text { developed in } \\
\text { this paper }\end{array}$ & 0 & 11,20 & 18,80 \\
\hline
\end{tabular}

The comparison of the results obtained using developed method with the results of FEM analysis, taken 
from the reference [24] for the same numerical example, are provided in Tabs. 1 and 2 for loading cases I and II, respectively.

The results given in Tabs. 1 and 2 clearly demonstrate that differences in bending-moments determined by FEM and the method given in this paper are negligible, even though the results were obtained using different approaches. By assuming that the member is homogenous, isotropic and ideally elastic, with constant prismatic crosssection, which is a very common case in construction, the calculation of constants and bending moments of semirigidly connected member are easily obtained by calculating the corresponding constants and bending moments at ends of member with ideally rigid connections and their subsequent "softening" with corresponding coefficients derived in section 4 .

Furthermore, the developed method with semi-rigid connections includes the previously existent deformation method with ideal connections, as its special case, which makes the method given in this paper more general and comprehensive.

\section{CONCLUSION}

Based on the results of many researches on the behaviour of real connections in steel frame structures that are available in the literature, it could be concluded that many advantages come with the implementation of structural analysis methods in which the connections are considered as deformable, i.e. semi-rigid. These methods enable more realistic modelling of the behaviour of the connections between structural elements. Their utilization in the design of steel frame structures can lead to significant reduction of material costs as well as in some reductions of the fabrication costs.

In this paper, a method was developed for the structural analysis of frame structures with semi-rigid connections. The developed method represents the extension of the existing method of deformation. The parameter that was implemented for the consideration of the degree of rigidity of connections was its rotational rigidity, which can be determined from experimental results or by using the procedures already implemented in structural design codes, such as EC3. The proposed method is relatively simple and convenient for both computational utilization and hand calculations, which is of great importance for its implementation in engineering practice.

The validation of the method was conducted by applying it on the example of simple frame structure and comparing the results with the results of FEM analysis. It was shown that the differences in bending-moments determined by FEM and the method given in this paper were negligible.

\section{REFERENCES}

[1] Abu-yasein, O. A. \& Frederick, G. R. (1994). Analysis of frames with semi-rigid joints. Computers and Structures, 52(6), 1161-1168. https://doi.org/10.1016/0045-7949(94)90182-1

[2] Arul Jayachandran, S., Marimuthu, V., Prabha, P., Seetharaman, S., \& Pandian, N. (2009). Investigations on the behaviour of semi-rigid endplate connections. Advanced Steel Construction, 5(4), 432-451.
https://doi.org/10.18057/IJASC.2009.5.4.5

[3] Baniotopoulos, C. C. \& Wald, F. (2000). The Paramount Role of Joints into the Reliable Response of Structures. Springer Netherlands. https://doi.org/10.1007/978-94-010-0950-8

[4] Bjorhovde, R. \& Colson, A. (1991). Economy of semi-rigid frame design. Connections in Steel Structures II. In Proceedings of the 2nd International Workshop, 418-429.

[5] Chan, S. L. \& Chui, P. P. T. (2000). Non-Linear Static and Cyclic Analysis of Steel Frames with Semi-rigid Connections. Oxford: Elsevier Science Ltd.

[6] Chen, W. F. (2000). Practical Analysis for Semi-Rigid Frame Design. Singapoore: World Scientific Publishing Co. https://doi.org/10.1142/4277

[7] Chen, W. F. (2008). Advanced analysis for structural steel building design. Frontiers of Architecture and Civil Engineering in China, 2(3), 189-196. https://doi.org/10.1007/s11709-008-0024-8

[8] Choi, S. \& Kim, S. (2006). Optimal Design of Semi-Rigid Steel Frames using Practical Nonlinear Inelastic Analysis, 6, 141-152.

[9] Đurić, M. (1962). Metoda deformacije. Zbornik Građevinskog Fakulteta Univerziteta u Beogradu, 5, 131161

[10] ECCS. Technical committee 8, Strutural stability, Analysis and Design of Steel Frames with Semi-rigid Joints. (1992).

[11] Elvin, A. \& Strydom, J. (2018). Optimizing Structures with Semi-Rigid Connections Using the Principle of Virtual Work. International Journal of Steel Structures, 18(3), 10061017. https://doi.org/10.1007/s13296-018-0043-9

[12] Eurocode 3: Design of steel structures - Part 1-8: Design of joints, 2005. (n.d.).

[13] Farkas, J., Jármai, K., \& Visser-Uys, P. (2003). Cost comparison of bolted and welded frame joints. Welding in the World, 47(1-2), 12-18. https://doi.org/10.1007/BF03266373

[14] Hadianfard, M. A. \& Razani, R. (2003). Effects of semi-rigid behavior of connections in the reliability of steel frames. Structural Safety, 25(2), 123-138. https://doi.org/10.1016/S0167-4730(02)00046-2

[15] Hwa, K. (2003). Toward Advanced Analysis in Steel Frame Design. Dissertation for Doctoral Degree. University of Hawaii.

[16] Jansen, W. \& Maatje, F. (1988). Calculation of the costs of steel structures with help of the computer (in Dutch). The Netherlands.

[17] Kartal, M. E., Başağa, H. B., Bayraktar, A., \& Muvafik, M. (2010). Effects of semi-rigid connection on structural responses. Electronic Journal of Structural Engineering, 10(January), 22-35.

[18] McGuire, W. (1992). Computer-aided analysis. In P. J. Dowling, J. E. Harding, \& R. Bjorhovde (Eds.), Constructional Steel Design an International Guide, 915935). New York: Elsevier.

[19] Patodi, S. C. \& Chauhan, J. M. (2011). First Order Analysis of Plane Frames with Semi-rigid Connections. National Conference on Recent Trends in Engineering \& Technology, (May).

[20] Plumier, A. (1994). Behaviour of connections. Journal of Constructional Steel Research, 29(1-3), 95-119. https://doi.org/10.1016/0143-974X(94)90058-2

[21] Sekulovic, M. \& Salatic, R. (2001). Nonlinear analysis of frames with flexible connections. Computers and Structures, 79(11), 1097-1107. https://doi.org/10.1016/S0045-7949(01)00004-9

[22] Weynand, K., Jaspart, J. P., \& Steenhuis, M. (1998). Economy studies of steel building frames with semi-rigid joints. Journal of Constructional Steel Research. https://doi.org/10.1016/S0143-974X(98)00045-5 
[23] Živković, S. (2009). Contribution to the calculation of steelframed constructions with semi-rigid connections (in Serbian). University of Niš.

[24] Živković, S. (2015). Contribution to the calculation of directly-welded connections in truss elements with hollow steel rectangular and square profiles, in Serbian, PhD Thesis. University of Niš.

[25] Zlatkov, D., Zdravkovic, S., Mladenovic, B., \& Stojic, R. (2012). Matrix formulation of dynamic design of structures with semi-rigid connections. Facta Universitatis - Series: Architecture and Civil Engineering, 9(1), 89-104. https://doi.org/10.2298/FUACE1101089Z

\section{Contact information:}

Srđan ŽIVKOVIĆ, assistant professor, PhD

(Corresponding author)

Faculty of Civil Engineering and Architecture,

University of Niš,

Aleksandra Medvedeva 14, 18000 Niš, Serbia

E-mail: srdjan.zivkovic@gaf.ni.ac.rs

Nenad STOJKOVIĆ, senior lecturer, PhD

Head of the Civil Engineering Department,

College of Applied Technical Sciences Niš

Aleksandra Medvedeva 20, 18000 Niš, Serbia

E-mail: svnenad@yahoo.com

Marija SPASOJEVIĆ-šURDILOVIĆ, assistant professor, PhD

Faculty of Civil Engineering and Architecture

University of Niš,

Aleksandra Medvedeva 14, 18000 Niš, Serbia

E-mail: spasojevic.n.marija@gmail.com

Marko MILOŠEVIĆ, Master in Civil Engineering, PhD student Innovation Center University of Nišs,

Faculty of Civil Engineering and Architecture,

University of Niš,

Univerzitetski trg 2, 18000 Niš, Serbia

E-mail: marko.milosevic@gaf.ni.ac.rs 